\title{
COMPRESSED SENSING FOR SYNTHETIC APERTURE RADAR IMAGING
}

\author{
Vishal M. Patel, Glenn R. Easley, Dennis M. Healy, Jr., and Rama Chellappa \\ University of Maryland, \\ College Park, MD 20742 \\ \{pvishalm@umd,geasley@umd,dhealy@umd,rama@cfar.umd\}.edu
}

\begin{abstract}
In this paper, we introduce a new Synthetic Aperture Radar (SAR) imaging modality that provides a high resolution map of the spatial distribution of targets and terrain based on a significant reduction in the number of transmitted and/or received electromagnetic waveforms. This new imaging scheme, which requires no new hardware components, allows the aperture to be compressed and presents many important applications and advantages among which include resolving ambiguities, strong resistance to countermesasures and interception, and reduced on-board storage constraints.
\end{abstract}

Index Terms - compressed sensing, SAR

\section{INTRODUCTION}

SAR is a radar imaging technology that is capable of producing high resolution images of the stationary surface targets and terrain. The main advantages of SAR are its ability to operate at night and in all weather conditions, hence overcoming limitations of both optical and infrared systems. There are four common modes of SAR: scan, stripmap, spotlight and inverse SAR (ISAR). In this paper, we will mainly focus on the spotlight mode SAR and ISAR. In spotlight mode SAR, the radar sensor steers its antenna beam to continuously illuminate the terrain patch being imaged. It can provide higher resolution than the stripmap and scan mode SAR because it maps a scene at multiple viewing angles during a single pass [1]. In ISAR, the radar is stationary and the target is moving. The angular motion of the target with respect to the radar can be used to form an image of the target. Differential Doppler shifts of adjacent scatters on a target are observed and the target's reflectivity function is obtained through the Doppler frequency spectrum [2].

Since a SAR image is a map of the spatial distribution of the reflectivity function of stationary targets and terrain, many SAR images can be sparse or compressible in some representation such as those from a wavelet or a complex wavelet transform. The recently introduced theory of compressed sensing (CS) states that it is possible to recover such sparse images from a small number of random measurements provided that the undersampling results in noise like artifacts in the transform domain and an appropriate nonlinear recovery scheme is used $[3,4]$.

In this paper, we introduce a new SAR image formation algorithm based on the theory of CS that reduces the number of transmitted and/or received waveforms. We will demonstrate that if the SAR image is assumed to be sparse in some transform domain, then one can reconstruct a good estimate of the reflectivity profile using this new image formation algorithm that relies on using a far fewer number of waveforms than the conventional systems do and requires no changes to a radar system hardware to work.

Our approach is in contrast to other compressive radar related algorithms that have only considered using CS as part of the analog-to-information (A2I) conversion [5] or transmitting specially designed waveforms [6]. It is also the case, that the radar community has considered similar concepts that we are presenting such as that provided in [7]. Yet our method enhances some of these suggestions and provides a proper framework along with general reconstruction techniques. By using concepts provided by CS-theory we are able to propose a reliable imaging system that should pave the way for many new applications that are highly desirable. Also, very recently CS ideas were applied for SAR image formation in [8].

\section{SAR PHASE HISTORIES}

In this section, we give a brief description of SAR [1, 9, 10, 11, 12]. Figure 1 shows a simple diagram of spotlight mode SAR and how received data are placed in a $2 \mathrm{D}$ signal array. In SAR, motion of the antenna is used to synthesize a long antenna. As the radar platform advances along its path at constant velocity, pulses are transmitted and received by the radar. Pulses are transmitted at an uniform pulse repetition interval (PRI), which is defined as $1 / P R F$, where PRF is the pulse repetition frequency. The reflected signal at any instant can be modelled as a convolution of the pulse waveform with the ground reflectivity function $[9,11]$.

It has been shown that, if the echo signal is mixed with the reference chirp signal and low-pass filtered, the resulting signal can be viewed as an approximate Fourier transform of the projection of the ground patch [11]. Consequently, the preprocessed SAR histories define a set of samples in the Fourier space ( $k$-space) of the scene on a polar wedge $[1,11,12]$. A reflectivity function (i.e. SAR image) is recovered by appropriately interpolating these polar data to a Cartesian grid and taking a two dimensional inverse Fourier transform. This algorithm is commanly known as the Polar Format Algorithm (PFA) [1].

The two dimensional image formed is interpreted in the dimensions of range and cross-range or azimuth. The range is the direction of signal propagation and the cross-range is the direction parallel to the flight path. Sometimes the range and the cross-range samples are referred to as the fast-time and the slow-time samples, respectively. 

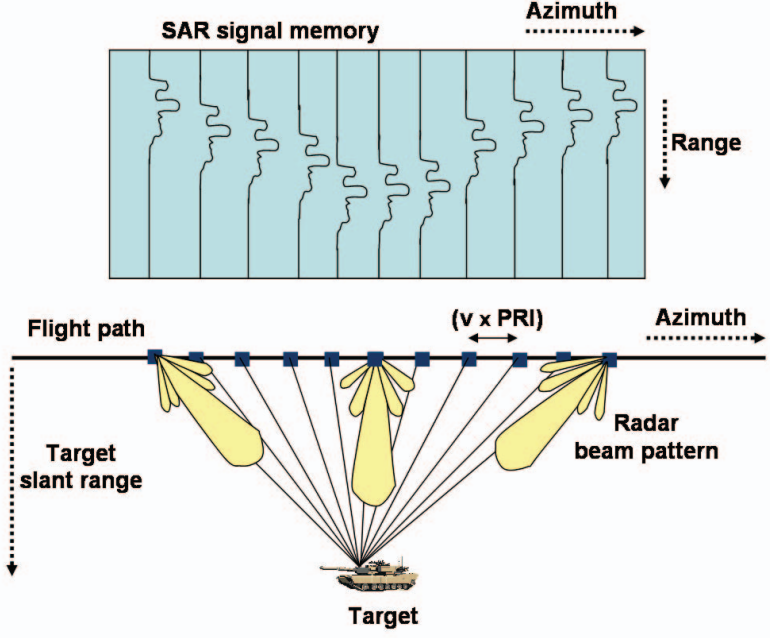

Figure 1: Spotlight SAR data collection in 2D.

\section{COMPRESSED SENSING}

A new framework known as compressed sensing, enables the reconstruction of sparse or compressible signals from a small set of nonadaptive, linear measurements. If properly chosen, the number of measurements can be much smaller than the number of Nyquist rate samples $[3,4]$. In this section, we give a brief introduction to compressed sensing.

Suppose $x \in \mathbb{C}^{N}$ is $K$-sparse in a basis or more generally a frame $\Psi$, so that $x=\Psi x_{0}$, with $\left\|x_{0}\right\|_{0}=K \ll N$, where $\|$. $\|_{0}$ returns the number of nonzero elements. In the case when $x$ is compressible in $\Psi$, it can be well approximated by the best $K$-term representation. Consider a random $M \times N$ measurement matrix $\Phi$ with $M<N$ and assume that $M$ linear measurements are made such that $y=\Phi x=\Phi \Psi x_{0}=\Theta x_{0}$. According to the theory of CS, when the matrix $\Theta$ has the Restricted Isometry Property (RIP) [20], it is possible to reconstruct $x$ from a set of $M=O\left(K \log \left(\frac{N}{K}\right)\right)$ linear measurements. A matrix $\Theta$ is said to satisfy the RIP of order $K$ with constants $\delta=\delta_{K} \in(0,1)$ if

$$
\left(1-\delta_{K}\right)\|v\|_{2}^{2} \leq\|\Theta v\|_{2}^{2} \leq\left(1+\delta_{K}\right)\|v\|_{2}^{2}
$$

for any $v$ such that $\|v\|_{0} \leq K$. A related condition requires that the rows of $\Phi$ cannot sparsely represent the columns of $\Psi$ and vice versa. In the case when $\Phi$ is generated from iid Gaussian distribution and $\Psi$ is a sparsifying basis, then $\Theta$ satisfies the RIP with high probability. Among many other ensembles of random matrices that satisfy the RIP, a partial Fourier matrix where $M$ rows of the $N \times N$ Fourier matrix selected at random are included.

When $\Theta$ has the RIP, it is possible to reconstruct $x$ via its coefficients $x_{0}$, by solving the following $\ell^{1}$ minimization problem $[3,4]$

$$
\hat{x_{0}}=\arg \min _{x_{0}^{\prime} \in \mathbb{R}^{N}}\left\|x_{0}^{\prime}\right\|_{1} \quad \text { subject to } y=\Phi \Psi x_{0}^{\prime} .
$$

The optimization problem (2) is often known as Basis Pursuit (BP) [13]. In the case when there are noisy observations, Basis Pursuit De-Noising (BPDN) can be used to estimate the original image.

\subsection{Compressive Sampling for SAR}

Design of a CS undersampling scheme for SAR entails the selection of phase histories such that it satisfies (1). Some of the results about CS are based on the fact that the Fourier samples are obtained randomly. However, sampling a truly random subset of the phase histories in SAR is usually impractical for existing hardware. In this section, we consider two compressed sensing $k$-space undersampling schemes for SAR. Since, the PRF essentially determines the slowtime-sampling rate, our CS undersampling schemes are based on modifying the PRF of the radar. Implementation of such undersampling schemes is very simple and requires a minor change to the PRF scheduling of the radar. More analysis on the aliasing artifacts introduce by these undersampling schemes in terms of the point spread function (PSF) will be discussed elsewhere (see [14] and [15] for more details).

Random slow-time undersampling: As discussed earlier, as the sensor advances along its path, pulses are transmitted and received by the radar (see Figure 1). The pulses are transmitted at every $P R I=\frac{1}{P R F}$. Undersampling methods applying regular grid produce regularly spaced strong aliases. Random placement of PRI can break up the periodicity of the aliasing artifacts and can convert strong aliases to random noise like artifacts $[16,17]$. For this reason, instead of transmitting pulses at every PRI, we propose to transmit a few pulses at a random PRI, which amounts to undersampling the 2D signal along the slow-time axis randomly.

Jittered slow-time undersampling: Jittered undersampling is based on a regular undersampling which is perturbed slightly by random noise. The effect of jitter in one dimension was analyzed by Balakrishnan in [18]. He analyzed time jitter in which the $n$th sample is jittered by an amount $\zeta_{n}$ so that it occurs at time $n P+\zeta_{n}$, where $P$ is the sampling period. He reported that if the $\zeta_{n}$ are uncorrelated then the following happens: high frequencies are attenuated, the energy lost to the attenuation appears as uniform noise, and the basic structure of the spectrum does not change $[16,17,18]$. Furthermore, in [19] it was shown that additive random jitter can eliminate aliasing completely. Jittered sampling in 2D was generalized and applied in computer graphics in [16, 17]. Also, Hennenfent and Herrmann in [15] have successfully applied 2D jittered undersampling in the context of CS for seismic data processing. Inspired by the properties of the jittered sampling, we propose to apply jitter undersampling in slow-time as well.

\section{SAR USING CS}

Given the partial Fourier measurements of the reflectivity map to be imaged, in this section, we show how CS can be used to reconstruct the SAR image.

Let $\sigma \in \mathbb{C}^{l \times l}$ be the reflectivity map to be imaged. Let $\Omega$ be some chosen set of frequencies of size $|\Omega|=M$, with $M \ll$ $l^{2}$, and $F_{\Omega}: \mathbb{C}^{l \times l} \rightarrow \mathbb{C}^{M}$ denote the partial Fourier transform operator restricted to $\Omega$. Let $S$ denote the collection of 2D phase histories and $\tilde{S}=F_{\Omega} \rho \in \mathbb{C}^{M}$ represent the collection of phase histories restricted to $\Omega$ (i.e. partial Fourier measurements of the reflectivity function $\rho$ obtained by incorporating one of the slowtime undersampling schemes discussed above). Assume that $\rho$ has a sparse representation (or is compressible) in some basis $\Psi$, so that $\rho=\Psi v$. Furthermore, assume that we are given the partial $k$-space noisy observations. Then, the reflectivity map $\rho$ can be recovered via $v$ by solving the following $\ell^{1}$ minimization problem

$$
v_{r e c}=\arg \min _{v^{\prime}}\left\|v^{\prime}\right\|_{1} \quad \text { s. t. } \quad\left\|\tilde{S}-F_{\Omega} \Psi v^{\prime}\right\|_{2} \leq \varepsilon,
$$


where $\varepsilon$ is a noise statistic that controls the fidelity of the reconstruction $[3,4,20]$. It was shown in [20], that the solution to (3) will recover the unknown sparse reflectivity map with an error proportional to the noise level. That is, $\left\|v-v_{\text {rec }}\right\|_{2} \leq C \varepsilon$, where the constant $C$ depends on the restricted isometry constant. Note that the operator, $F_{\Omega}$, can be a non-uniform Fourier transform operator $[21,22]$. Implementation of such a scheme requires a reformulation that includes an operator that maps the phase history data into the image domain [12].

\section{SAR AND ISAR EXAMPLES}

In this section, we demonstrate the performance and applicability of our compressive imaging algorithm on synthetic and real SAR data. In the first example, we used four point targets to generate the phase histories. We used the following parameters in our simulation: center frequency $=3.80 \times 10^{9} \mathrm{~Hz}$, synthetic aperture length $=102 \mathrm{~m}$, bandwidth $=1.35 \times 10^{8} \mathrm{~Hz}, \mathrm{~A} / \mathrm{D}$ complex sampling rate $=1.85 \times 10^{8} \mathrm{~Hz}$, and transmitted pulse length $=$ $1 \times 10^{-6}$. Figure 2 (a) shows the reconstruction of the SAR image from the full simulated data using the PFA [1]. In Figure 2(b), we show the phase histories obtained after applying the random slow-time undersampling. Only $30 \%$ of the data was used. Figure 2 (c), shows the traditional reconstruction from the compressive measurements by the PFA, which fails to reconstruct the image. Figure 2(d) shows the reconstructed image using our proposed method. We choose $\Psi$ to be the identity matrix for this example. Our reconstruction used the spectral projected gradient (SPGL1) algorithm [23]. As can be seen from Figure 2(d), that we were able to image the reflectivity profile of the point targets as good as PFA did from the full measurement data. Furthermore, we use the Peak Sidelobe Ratio (PSR) and the Integrated Sidelobe Ratio (ISLR) as measures of performance to evaluate the reconstructed point targets. The PSR determines the difference between the mainlobe and the most prominent sidelobe. The ISLR measures the ratio of all energy in the mainlobe to the total energy in all the sidelobes [1].

\begin{tabular}{|c|c|c|c|c|}
\hline & Origina & & $\mathrm{CS}$ & \\
\hline & PSR & ISLR & PSR & ISLR \\
\hline Range & -29.64 & -23.31 & $\overline{-29.63}$ & -23.33 \\
\hline Cross-range & -35.5 & -28.29 & -34.90 & -27.94 \\
\hline
\end{tabular}

We used a point target located at the top in the image for comparison. Since the reconstructed point response is a $2 \mathrm{D}$ entity, we used principle axis cuts namely the range-cut and the cross-rangecut for the analysis. We will refer to the range-cut and the corssrange-cut as the range impulse response (RIR) and the cross-range impulse response (CRIR), respectively. There is no degradation in PSR and ISLR for the RIR. However, there is a minor degradation in the CRIR due to the fact that our 2D undersampling scheme is along the cross-range axis.

In the second experiment, we used ISAR data collected on a SAAB 9000 car using System Planning Corporation's Mark V radar $^{1}$. We reconstructed the image after $60 \%$ jittered slow-time undersampling was applied to the data. As can be seen from Figure 3 , the reconstructed image from these compressed measurements is identical to the one reconstructed from the full measure-

\footnotetext{
${ }^{1}$ specifications of the radar can be found at www.sysplan.com/Radar
}

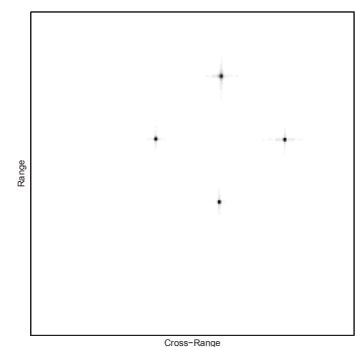

(a)

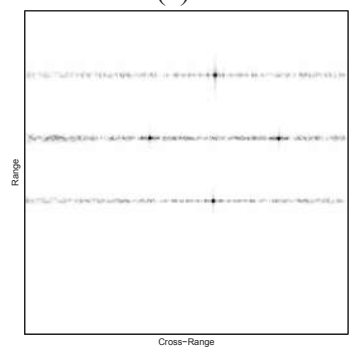

(c)

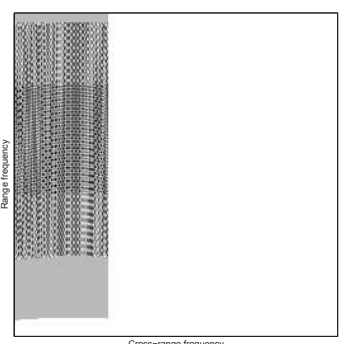

(b)

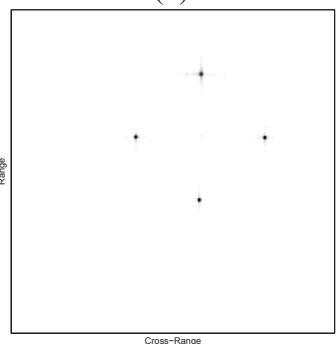

(d)
Figure 2: Point targets example. (a) Traditional PFA based reconstruction from the full simulated data. (b) Random slow-time undersampled phase histories. (c) Reconstructed by the PFA from the compressive measurements. (d) Reconstructed image using our method.

ments. Figure 3(b), shows how the traditional reconstruction fails to recover the ISAR image.

In $[4,3]$, a theoretical bound on the number of Fourier samples that need to be measured for a good reconstruction has been derived. However, it has been observed by many researchers [14, $20,24,25]$, that in practice Fourier samples in the order of two to five times the number of sparse coefficients suffice for a good reconstruction. Our experiments also support this claim.

\section{APPLICATIONS}

The idea of transmitting waveforms at a non-uniform PRI for SAR has been suggested before [7]. This method, however, suffers from the smearing of the image in the cross-range dimension due to the randomness of the PRI. By solving the basis pursuit denoising problem (i.e (3)), we are able to not only reconstruct the image as good as some of the traditional SAR reconstruction methods do from the full data but even at a lower sampling rate. Our method of compressing the synthetic aperture offers many advantages:

- Since, irregularly transmitted waveforms are much more harder to intercept, our compressive aperture method offers strong countermeasures resistance [7, 26, 27].

- Many methods for resolving ambiguities have been suggested. Among them include pulse tagging, PRF switching, and methods based on the Chinese remainder theorem. PRF switching is a simple extension of PRF jittering. Thus, our compressive radar can also be useful in resolving ambiguities [28].

- In many SAR systems, radar data is directly stored in a memory for later transmission to the ground. Our compressive SAR has the potential to significantly reduce the amount of data to be stored and transmitted $[9,29]$. 


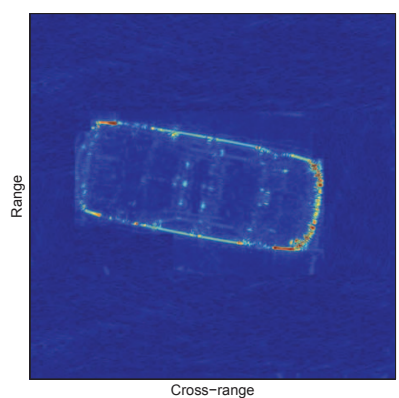

(a)

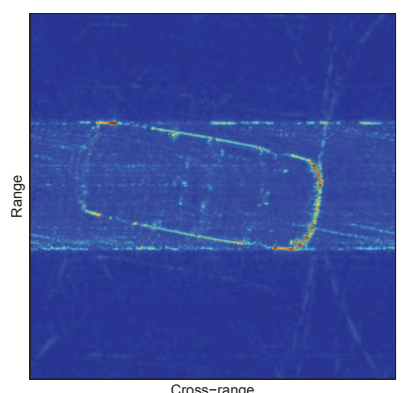

(b)

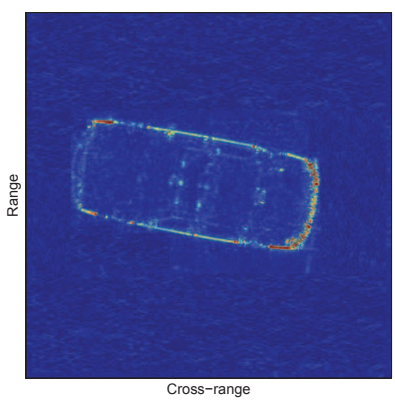

(c)

Figure 3: SAAB car ISAR example. (a) Traditional reconstruction with the full data. (b) Traditional reconstruction with the partial data. (c) Reconstructed image using our proposed method from $60 \%$ slow-time undersampled data.

\section{CONCLUSION}

We have managed to utilize CS-theory and demonstrated that it is possible to compress the synthetic aperture for radar imaging. Most importantly, not only can our suggested undersampling be used in novel collection schemes to produce high quality images but many new applications such as signals intelligence, resolving ambiguities, and reduced storage constraints are possible because of it.

\section{REFERENCES}

[1] W. G. Carrara, R. S. Goodman, and R. M. Majewski, Spotlight Synthetic Aperture Radar: Signal Processing Algorithms. Norwood, MA, Artech House, 1995.

[2] V. C. Chen and H. Ling, Time-Frequency Transforms for Radar Imaging and Signal Analysis. Norwood, MA, Artech House, 2002.

[3] D. Donoho, "Compressed sensing," IEEE Trans. Info. Theory, vol. 52, no. 4, pp. 1289-1306, April 2006.

[4] E. Candes, J. Romberg and T. Tao, "Robust Uncertainty Principles: Exact Signal Reconstruction from Highly incomplete Frequency Information," IEEE Trans. Info. Theory, vol. 52, no. 2, pp. 489509, Feb. 2006.

[5] R. Baraniuk and P. Steeghs, "Compressive radar imaging, Proc. 2007 IEEE Radar Conf., pp. 128133, Apr. 2007.

[6] M. Herman and T. Strohmer. "High resolution radar via compressed sensing", To appear in IEEE Trans. Signal Processing.

[7] S.G. Mobley, M.W. Maier, "Synthetic aperture radar with a nonuniform pulse repetition interval," ssst, pp. 498-502, 27th Southeastern Symposium on System Theory (SSST’95), 1995.

[8] I. Stojanovic, W. C. Karl and M. Çetin, "Compressed sensing of mono-static and multi-static SAR, "Proc. SPIE, vol. 7337, 2009.

[9] I. G. Cumming, and F. H. Wong, Digital processing of synthetic aperture radar data. Norwood, MA, Artech House, 2005.

[10] K. Tomiyasu, "Tutorial review of Synthetic-Aperture Radar (SAR) with applications to imaging of the ocean surface, "Proc. of the IEEE, vol. 66, no. 5, pp. 563-583, May 1978.

[11] D. C. Munson,Jr., J. D. O'Brien, and W. K. Jenkins, "A tomographic formulation of spotlight-mode synthetic aperture radar," Proc. IEEE, vol. PROC-71, pp. 917-925, Aug. 1983.

[12] M. Çetin and W. C. Karl, "Feature-enhanced synthetic aperture radar image formation based on nonquadratic regularization," IEEE Trans. Image Process., vol. 10, no. 4, pp. 623-631, Apr. 2001.
[13] S. Chen, D. Donoho, and M. Saunders, “ Atomic Decomposition by Basis Pursuit," SIAM J. Sci. Comp., vol. 20, no. 1, pp. 33-61, 1998.

[14] M. Lustig, D. Donoho, and J. M. Pauly, "Sparse MRI: The Application of Compressed Sensing for Rapid MR Imaging," Magnetic Resonance in Medicine,vol. 58, no. 6, pp. 1182-1195, Dec. 2007.

[15] G. Hennenfent and F. J. Herrmann. "Simply denoise: wavefield reconstruction via jittered undersampling", Geophysics, vol. 73, no. 3, pp. v19-v28, June 2008.

[16] R. L. Cook, " Stochastic sampling in computer graphics," $A C M$ Transactions on Graphics, vol. 5, no. 1, pp. 51-72, Apr. 1986.

[17] M. Dippe, and E. Wold, "Antialiasing through stochastic sampling,” SIGGRAPH, vol. 19, no. 3, pp. 69-78, Nov. 1985.

[18] A. V. Balakrishnan, " On the problem of time jitter in sampling," IRE Trans. Inf. Theory, vol. 8, no. 3, pp. 226-236, Apr. 1962.

[19] H. S. Shapiro, and R. A. Silverman, " Alias-free sampling of random noise," SIAM, vol. 8, no. 2, pp. 225-248, June 1959.

[20] E. Candes, J. Romberg and T. Tao, "Satble signal recovery from incomplete and inaccurate measurements," Communications in Pure and Applied Mathematics, vol. 59, pp. 1207-1223, 2006.

[21] J. A. Fessler and B. P. Sutton, "Nonuniform fast Fourier transforms using min-max interpolation," IEEE Trans. Sig. Proc., vol. 51, no. 2, pp. 560-574, Feb. 2003.

[22] M. M. Bronstein,A. M. Bronstein, M. Zibulevsky and H. Azhari, "Reconstruction in diffraction ultrasound tomography using nonuniform FFT, " IEEE Trans. Medical Imag., vol. 21, no. 11, pp. 1395-1401, Nov. 2002.

[23] E. van den Berg and M. P. Friedlander, "SPGL1: A solver for largescale sparse reconstruction", http://www.cs.ubc.ca/labs/scl/spgl1/.

[24] E. Candes and J. Romberg, "Signal recovery from random projections," in Proc. of SPIE Computational Imaging III, San Jose, vol. 5674, 2005.

[25] Y. Tsaig and D. L. Donoho, "Extensions of compressed sensing," Signal Processing, vol. 86, no. 3, pp. 533-548, March 2006.

[26] W. W. Goj, Synthetic Aperture Radar and Electronic Warfare, Artech House, 1992.

[27] R. J. Anderson, Security Engineering: A Guide to Building Dependable Distributed Systems. Indianapolis, IN, Wiley, 2008.

[28] G. E. Stimson, Introduction to airborne radar. second edition, Mendham, NJ, SciTech Publishing, Inc., 1998.

[29] S. Bhattacharya, T. Blumensath, B. Mulgrew and M. Davis, "Fast encoding of synthetic aperture radar raw data using compressed sensing," IEEE wrokshop on SSP, Aug. 26-29 2007. 